\title{
QUALIDADE DO SOLO NA CULTURA DO CAJUEIRO ANÃO PRECOCE CULTIVADO SOB DIFERENTES SISTEMAS DE MANEJO ${ }^{(1)}$
}

\author{
Kátia Araújo Ribeiro ${ }^{(2)}$, Teógenes Senna de Oliveira ${ }^{(3)}$, Eduardo de Sá \\ Mendonça ${ }^{(4)}$, Francisco Alisson da Silva Xavier ${ }^{(5)}$, Stoécio Malta \\ Ferreira Maia $^{(6)}$ \& Helon Hébano de Freitas Sousa ${ }^{(7)}$
}

\begin{abstract}
RESUMO
O Brasil é o principal exportador de castanha de caju, estando a maioria de sua produção concentrada no Nordeste $(97 \%)$. Todavia, essa produção tem sido reduzida nos últimos anos, sobretudo no Estado do Ceará, especialmente devido ao uso inadequado do solo. Objetivou-se avaliar as alterações nas propriedades físicas e químicas de um Argissolo Acinzentado e o desenvolvimento das plantas de cajueiro anão em experimento de campo com diferentes sistemas de manejo do solo. Foram testados os seguintes sistemas: gradagem + coroamento, roçagem mecânica + coroamento, roçagem manual + coroamento, cobertura vegetal + coroamento, cobertura vegetal + cobertura morta e uso de herbicida. O delineamento experimental utilizado foi o de blocos casualizados com parcelas subdivididas, considerando-se os diferentes sistemas de manejo e as profundidades de amostragem do solo como parcelas e subparcelas, respectivamente, e três repetições. Foram avaliadas as características e, ou, propriedades físicas (granulometria, densidade do solo, densidade de partículas, resistência à penetração, condutividade hidráulica, porosidade) e químicas (cátions trocáveis, pH, matéria orgânica e fracionamento de fósforo) do solo, a produtividade da castanha de caju, a altura e o diâmetro de copa das plantas de cajueiro durante o período de 1999 a 2002 . Os sistemas de manejo estudados promoveram alterações nas propriedades químicas do solo, principalmente naquelas associadas à sua matéria orgânica, com reflexos positivos nos sistemas que utilizam o herbicida e a
\end{abstract}

\footnotetext{
(1) Parte da Tese de Mestrado do primeiro autor apresentada ao Departamento de Ciências do Solo da Universidade Federal do Ceará - UFC. Executado com recursos da CAPES e FUNCAP. Recebido para publicação em agosto de 2005 e aprovado em fevereiro de 2007.

(2) Engenheiro-Agrônomo, M.Sc. Departamento de Ciências do Solo, Universidade Federal do Ceará - UFC. Campus do Pici, CEP 60455-760 Fortaleza (CE). E-mail: katribeiro@yahoo.com.br

(3) Professor Adjunto do Departamento de Ciências do Solo, UFC. Bolsista do CNPq. E-mail: teo@ufc.br

(4) Professor Adjunto do Departamento de Solos, Universidade Federal de Viçosa - UFV. CEP 36570-000 Viçosa (MG). Bolsista do CNPq. E-mail: esm@ufv.br

(5) Doutorando do Departamento de Solos, UFV. E-mail: ali_xavier@yahoo.com.br

(6) Doutorando da Escola Superior de Agricultura Luiz de Queiroz, Universidade de São Paulo - ESALQ/USP. Av. Pádua Dias 11, CEP 13418-900 Piracicaba (SP). E-mail: stoecio@hotmail.com

(7) Mestrando do Departamento de Ciências do Solo, UFC. E-mail: helonhebano@yahoo.com.br
} 


\begin{abstract}
roçagem manual e negativos no que envolve a grade de discos. O não-revolvimento superficial do solo e a não-fragmentação da biomassa aérea das plantas espontâneas presentes nas entrelinhas, previstos nos sistemas que utilizam o herbicida e a roçagem manual, constituem-se em estratégias de manejo favoráveis à melhoria da qualidade do solo e à produtividade dos cajueiros. A ausência da prática do coroamento promoveu melhor qualidade do solo e maiores níveis de produtividade de castanha, em decorrência do aumento do aporte de matéria orgânica no solo e do não-revolvimento superficial deste, permitindo inferir que não se trata de uma prática adequada às culturas perenes.
\end{abstract}

Termos de indexação: fracionamento de $P$, coroamento, castanha de caju, solopropriedades físicas, solo-propriedades químicas.

\title{
SUMMARY: SOIL QUALITY IN SOIL MANAGEMENT SYSTEMS IN DWARF CASHEW CROPS
}

Brazil is the most important exporter of cashew nut in the world. The production is concentrated in the northeast region, which accounts for $97 \%$ of the national production. However, in the recent years the cashew nut production has been declining, specially in the state of Ceará, mainly due to inadequate soil management. The aim of this study was to evaluate alterations in the physical and chemical properties of an Ultisol submitted to distinct soil management practices and the development of dwarf cashew trees in a field experiment. The following soil management systems were evaluated: disc harrow + weeding, mechanic mowing + weeding, hand mowing + localized weeding, vegetal cover + localized weeding, vegetal cover + organic residue cover and herbicide use. A split-plot design arranged in randomized blocks was used, considering the different management systems as plots and soil depths as split-plots. Three replications were used. The following soil physical properties were analyzed: granulometry, bulk density, particle density, soil penetration resistance, hydraulic conductivity, soil porosity, as well as chemical properties (exchangeable elements, soil $\mathrm{pH}$, organic matter and phosphorus fractions), and cashew nut yield, plant height and canopy diameter from 1999 to 2002. The management systems that resulted in the most intense soil mobilization and fragmentation of biomass of cover plants (disc harrow, localized weeding and mechanical mowing) favored the degradation of physical and chemical properties, thus reducing the soil quality. Higher and more stable cashew nut yields were obtained in the systems where the practice of removing the vegetation around the trees (localized weeding) had been abandoned, resulting in higher organic matter inputs and absence of soil disturbance. The practice of localized weeding is therefore not recommendable for perennial crops.

Index terms: $P$ fractionation, soil quality, localized weeding, cashew nut, soil-physical properties, soil-chemical properties.

\section{INTRODUÇÃO}

Desde os primórdios de seus primeiros registros, por volta de 1558, durante a dominação francesa no Brasil, o cajueiro (Anacardium occidentale L.) era reconhecido como fonte preciosa de alimentação e utilidade para os índios e jesuítas (Lima, 1988). A importância da cajucultura no Brasil ressalta-se, principalmente, na região Nordeste, garantindo renda no período de entressafra das principais culturas exploradas na região, como milho e feijão (Lopes Neto, 1997).

Alguns autores têm destacado reduções significativas nos níveis de produção e na produtividade das áreas cultivadas com cajueiro. Segundo Pimentel et al. (1993), o rendimento médio por hectare na produção de castanha teve redução no período de 1978 a 1990 nos Estados do Ceará, Piauí e Rio Grande do Norte. Resultados de levantamentos realizados pelo IBGE revelaram que esses mesmos Estados obtiveram juntos, no ano de 1999, rendimento médio de castanha em torno de $219 \mathrm{~kg} \mathrm{ha}^{-1}$, sendo este reduzido para $171 \mathrm{~kg} \mathrm{ha}^{-1}$ no ano de 2001 (IBGE, 2004).

Alguns fatores estão relacionados com a queda nos níveis de produção dos cajueiro, dentre os quais os principais são: a baixa qualidade dos pomares cultivados, a baixa fertilidade dos solos nos quais esses pomares estão inseridos, o reduzido uso de insumos (adubos, corretivos, defensivos agrícolas, etc.) e o uso inadequado de práticas de manejo do solo (Araújo, 1990).

De modo geral, na condução dos pomares de cajueiro são adotadas práticas de manejo problemáticas 
para os solos cultivados. Como exemplo, tem-se a limpeza do material orgânico depositado sob a copa dos cajueiros, oriundo da queda de folhas e ramos, como também do material originado pelas plantas espontâneas ali presentes. Essa prática é conhecida regionalmente como "coroamento".

Nesse contexto, práticas alternativas de manejo, como adubação verde, manejo de restos de cultura e cobertura morta, são eficientes para o aumento da capacidade produtiva dos solos e da produção de material orgânico, minimizando o efeito da compactação e dos processos erosivos dos solos cultivados (Paula et al., 1998). Quanto ao aspecto físico do solo, Barreto \& Fernandes (2001) destacam o efeito de leguminosas no aumento da macroporosidade e na redução da densidade de solos de Tabuleiros Costeiros. Em adição, Monreal et al. (1997) observaram influência positiva da matéria orgânica sobre a proporção de agregados estáveis do solo, comprovando o benefício dela para a estrutura do solo. Da mesma forma, as características químicas, como teor de carbono orgânico, $\mathrm{pH}$ e cátions trocáveis, podem ser afetadas diretamente pelo manejo do solo (Cattelan \& Vidor, 1990). De modo geral, os sistemas de manejo sem revolvimento e que favorecem a adição de resíduos orgânicos aumentam o teor de carbono orgânico do solo (Testa et al., 1992; Bayer \& Mielniczuk,1997), embora esse aumento normalmente seja restrito às camadas superficiais (Bayer \& Mielniczuk, 1997). Algumas alternativas de uso do solo reduzem a utilização de máquinas e implementos e, conseqüentemente, os efeitos negativos sobre as propriedades e, ou, características dos solos, que comprometem a qualidade e produtividade das culturas (Leite et al., 2003).
O objetivo do presente estudo foi avaliar as alterações nas propriedades físicas e químicas de um Argissolo Acinzentado e no desenvolvimento das plantas de cajueiro anão precoce CCP 76 cultivadas sob diferentes sistemas de manejo, em Pacajus, Ceará.

\section{MATERIAL E MÉTODOS}

O experimento foi instalado no Campo Experimental de Pacajus, do Centro Nacional de Pesquisa da Agroindústria Tropical da Empresa Brasileira de Pesquisa Agropecuária (CNPATEmbrapa), situado no município de Pacajus-CE, a $50 \mathrm{~km}$ de Fortaleza (4 $10^{\prime} \mathrm{S}$ e $\left.38^{\circ} 27^{\prime} \mathrm{W}\right)$. As temperaturas médias anuais mínima e máxima da região estão em torno de 25 e $35^{\circ} \mathrm{C}$, respectivamente (IPLANCE, 2004). O solo da área foi classificado como Argissolo Acinzentado distrófico arênico A moderado de textura média fase caatinga litorânea relevo plano (Quadro 1).

O experimento iniciou-se em 1997, em área de aproximadamente 2 ha, onde foram plantadas mudas de cajueiro anão precoce clone CCP 76 no espaçamento de $7 \times 7 \mathrm{~m}$. Cada parcela experimental era composta de 16 plantas, considerando-se como parcela útil somente quatro delas. Ao longo dos anos foram realizadas adubações de manutenção, com doses variadas, conforme o estádio de desenvolvimento das plantas, efetuadas sempre na projeção da copa com incorporação ao solo. De 1998 a 2000, a adubação adotada por planta foi de 178, 300 e 100 g de uréia, $\mathrm{P}_{2} \mathrm{O}_{5}$ e $\mathrm{K}_{2} \mathrm{O}$, respectivamente. Em 2001, segundo a recomendação, as doses foram de 140, 100 e 120 g de

Quadro 1. Propriedades físicas e químicas de horizonte Bt1 de um Argissolo Acinzentado, na profundidade de 95-100 cm, cultivado com cajueiro anão precoce CCP 76, Pacajus-CE

\begin{tabular}{|c|c|c|c|}
\hline Propriedade & Resultado & Propriedade & Resultado \\
\hline pH em água & 4,40 & PST (\%) & 1,00 \\
\hline $\mathrm{Ca}^{2+}$ trocável $\left(\mathrm{cmol}_{\mathrm{c}} \mathrm{kg}^{-1}\right)$ & 0,60 & $\mathrm{C}\left(\mathrm{g} \mathrm{kg}^{-1}\right)$ & 2,22 \\
\hline $\mathrm{Mg}^{2+}$ trocável $\left(\mathrm{cmol}_{\mathrm{c}} \mathrm{kg}^{-1}\right)$ & 0,50 & $N\left(g_{k g}^{-1}\right)$ & 0,22 \\
\hline $\mathrm{Na}^{2+}$ trocável $\left(\mathrm{cmol}_{\mathrm{c}} \mathrm{kg}^{-1}\right)$ & 0,04 & Relação C/N & 10,00 \\
\hline $\mathrm{K}^{2+}$ trocável $\left(\mathrm{cmol}_{\mathrm{c}} \mathrm{kg}^{-1}\right)$ & 0,20 & Matéria Orgânica (g kg·-1) & 3,83 \\
\hline $\mathrm{H}^{+}+\mathrm{Al}^{3+}\left(\mathrm{cmol}_{\mathrm{c}} \mathrm{kg}^{-1}\right)$ & 1,05 & $\mathrm{P}$ disponível $\left(\mathrm{mg} \mathrm{kg}^{-1}\right)$ & 1,00 \\
\hline $\mathrm{Al}^{3+}$ trocável $\left(\mathrm{cmol}_{\mathrm{c}} \mathrm{kg}^{-1}\right)$ & 1,00 & Areia fina $\left(\mathrm{g} \mathrm{kg}^{-1}\right)$ & 190,00 \\
\hline $\mathrm{S}\left(\mathrm{cmol}_{\mathrm{c}} \mathrm{kg}^{-1}\right)$ & 1,30 & Areia grossa (g kg-1) & 530,00 \\
\hline $\mathrm{T}\left(\mathrm{cmol}_{\mathrm{c}} \mathrm{kg}^{-1}\right)$ & 3,10 & Silte $\left(\mathrm{g} \mathrm{kg}^{-1}\right)$ & 60,00 \\
\hline $\mathrm{V}(\%)$ & 42,00 & Argila $\left(\mathrm{g} \mathrm{kg}^{-1}\right)$ & 220,00 \\
\hline m (\%) & 49,00 & Densidade de partícula $\left(\mathrm{Mg} \mathrm{m}^{-3}\right)$ & 2,64 \\
\hline
\end{tabular}


uréia, $\mathrm{P}_{2} \mathrm{O}_{5}$ e $\mathrm{K}_{2} \mathrm{O}$, respectivamente. Já em 2002 a recomendação adotada foi modificada para 200,300 e $180 \mathrm{~g}$ de uréia, $\mathrm{P}_{2} \mathrm{O}_{5}$ e $\mathrm{K}_{2} \mathrm{O}$, respectivamente. O fertilizante fosfatado foi aplicado em dose única, enquanto os demais foram divididos em três dosagens, obedecendo a um intervalo mínimo de um mês entre elas. As adubações foram feitas geralmente nos meses de fevereiro e março ou no início do período chuvoso. As fontes utilizadas para $\mathrm{P}_{2} \mathrm{O}_{5}$ e $\mathrm{K}_{2} \mathrm{O}$ foram o superfosfato simples e o cloreto de potássio, respectivamente.

Os tratamentos consistiram dos seguintes sistemas de manejo:

a) Gradagem + coroamento (Grad): utilizou-se uma grade destorroadora de 10 discos de $60 \mathrm{~cm}$ de diâmetro, acoplada a trator agrícola de pneus. As plantas espontâneas eram trituradas e incorporadas à profundidade de $15 \mathrm{a} 20 \mathrm{~cm}$.

b) Roçagem mecânica + coroamento (Rmec): realizada com roçadeira mecânica acoplada a trator agrícola de pneus. As plantas espontâneas eram trituradas pelo implemento e deixadas sobre a superfície do solo.

c) Roçagem manual + coroamento (Rman): esta prática foi realizada manualmente, usando foices. O material vegetal era cortado próximo ao colo das plantas e deixado sobre a superfície.

d) Cobertura vegetal + coroamento (Cv): nas entrelinhas das plantas de cajueiro anão precoce foram cultivadas leguminosas visando à cobertura do solo, fixação e incorporação de $\mathrm{N}$ atmosférico. As leguminosas foram plantadas anualmente, no início do período chuvoso, após uma gradagem na superfície. As leguminosas utilizadas foram: calopogônio (Calogonium muconoides), em 1997 e 1998; cunhã (Clitoria ternaria), em 1999 e 2000; e guandu (Cajanus cajan), em 2001 e 2002. Ao final do período chuvoso estas plantas eram roçadas mecanicamente e deixadas sobre a superfície do solo.

e) Cobertura vegetal + cobertura morta $(\mathrm{Cv}+\mathrm{Cm})$ : este tratamento é semelhante ao anterior, porém, em substituição ao coroamento, usou-se a bagana de carnaúba (Copernicia cerifera L.) como cobertura morta. A quantidade de bagana foi de aproximadamente $15 \mathrm{~kg}$ por planta, espalhada na projeção da copa.

f) Herbicida (Herb): o manejo das plantas espontâneas nas entrelinhas foi realizado com a aplicação de herbicida sistêmico glyphosate, na dosagem de $3 \mathrm{~L}$ por hectare, diluídos em $400 \mathrm{~L}$ de água. A aplicação foi feita também sob a copa das plantas de cajueiro, conservando-se sobre o solo o material orgânico originado das plantas espontâneas ou mesmo das plantas de cajueiro.

À exceção dos sistemas $\mathrm{Cv}+\mathrm{Cm}$ e Herb, nos demais tratamentos realizou-se o coroamento, que consiste na remoção de resíduos orgânicos e plantas espontâneas existentes sob a copa das plantas de cajueiro. Essa prática foi realizada com o objetivo de facilitar a colheita das castanhas na projeção da copa dos cajueiros.

As amostras de solo foram coletadas nas entrelinhas, em diferentes épocas. Em abril de 2002 foram feitas coletas de amostras de solo em 11 camadas, considerando espessuras de 2,5 e $10 \mathrm{~cm}$, originando as seguintes camadas: $0-2,2-4,4-6,6-8$, 8-10, 10-15, 15-20, 20-30, 30-40, 40-50 e 50-60 cm. Por meio da análise de carbono orgânico total, foi feita a união de camadas, estabelecendo aquelas que foram utilizadas nas análises físicas e químicas do solo, sendo estas de $0-6,6-15,15-30$ e $30-60 \mathrm{~cm}$. Foram realizadas também coletas de amostras de solo nos anos de 1998 e 2000, considerando as profundidades de $0-20$ e $20-40 \mathrm{~cm}$. Nesse caso, as variáveis quantificadas nestes anos na camada de $0-20 \mathrm{~cm}$ foram comparadas à média das profundidades de 0-6 e 6$15 \mathrm{~cm}$ do ano de 2002. De modo semelhante, a camada de 20-40 cm foi comparada com a média das profundidades de 15-30 e 30-60 cm entre os anos relatados. As amostras deformadas foram secas ao ar (TFSA), destorroadas e peneiradas em malha de $2 \mathrm{~mm}$.

A densidade do solo (Ds) foi determinada pelo método do anel volumétrico, e a das partículas (Dp), pelo método do balão volumétrico. A porosidade total do solo (Pot) foi estimada pela relação entre a Ds e a Dp. A microporosidade (Mip) foi obtida pelo método da mesa de tensão, enquanto a macroporosidade (Map) foi calculada pela diferença entre a Pot e a Mip. A condutividade hidráulica foi determinada com saturação das amostras coletadas em cilindro de PVC em permeâmetro de carga constante. Todas essas análises foram realizadas conforme Embrapa (1997). Amostras de solo para análise de condutividade hidráulica $(\mathrm{CH})$ foram coletadas nas profundidades de 0-10 e 20-30 cm, sendo feita, também, uma média das duas profundidades anteriores para a expressão dos dados da camada de 0-30 cm.

A resistência à penetração foi avaliada em campo, utilizando-se penetrômetro de anel, modelo Soiltest CN-970, coletando-se amostras de solo para determinação da sua umidade, após secagem das amostras em estufa por $24 \mathrm{~h}$ a $105^{\circ} \mathrm{C}$.

Os ions $\mathrm{Ca}^{2+}, \mathrm{Mg}^{2+} \mathrm{e}^{3+}$ trocáveis foram extraídos com solução de $\mathrm{KCl} 1 \mathrm{~mol} \mathrm{~L}^{-1}$, sendo os dois primeiros elementos determinados por espectrofotômetro de absorção atômica e o último, por titulometria. O P e $\mathrm{K}$ disponíveis foram extraídos com Mehlich-1 e determinados por colorimetria e fotometria de chama, respectivamente. A acidez potencial $(\mathrm{H}+\mathrm{Al})$ foi extraída com solução de acetato de cálcio $0,5 \mathrm{~mol} \mathrm{~L}^{-1}$ a pH 7,0 e determinada por titulometria. $\mathrm{O} \mathrm{pH}$ em água foi determinado utilizando a relação solo:líquido de 1:2,5 (Embrapa, 1999). Os teores de soma de bases (S), capacidade de troca catiônica (T) e saturação por bases (V) foram estimados a partir dos resultados das análises anteriormente descritas. 
Os teores de matéria orgânica (MO) foram calculados com base nos teores de carbono orgânico total quantificado por oxidação da matéria orgânica via úmida, com fonte externa de calor (Yeomans \& Bremner, 1988).

$\mathrm{O}$ fracionamento de fósforo $(\mathrm{P})$ foi realizado conforme proposto por Bowman (1989), que preconiza a extração do elemento em meios ácido e básico. Primeiramente, foi extraído o P inorgânico (Pi) e, em seguida, os extratos foram submetidos à digestão perclórica $(\mathrm{HClO} 4)$, para extração de $\mathrm{P}$ total $(\mathrm{Pt})$. O $\mathrm{P}$ orgânico (Po), em meios ácido e básico, foi obtido por diferença entre o Pi e o Pt. A quantificação de P nos extratos foi realizada por colorimetria (Murphy \& Riley, 1962).

A produtividade da castanha de caju, a altura e os diâmetros de copa das plantas de cajueiro foram determinados nos anos de 1999, 2000, 2001 e 2002.

O delineamento experimental foi o de blocos casualizados com parcelas subdivididas ou subsubdivididas, considerando-se os diferentes sistemas de manejo, as profundidades de amostragem do solo e os anos de estudo como parcelas, subparcelas e subsubparcelas, respectivamente, sendo adotadas três repetições. Foi realizada a análise de variância e as médias comparadas pelo teste de Tukey a $5 \%$, utilizando-se o programa Sistema de Análises Estatísticas e Genéticas (SAEG), desenvolvido pela Universidade Federal de Viçosa.

\section{RESULTADOS E DISCUSSÃO}

\section{Propriedades físicas do solo}

A condutividade hidráulica $(\mathrm{CH})$, densidade de partículas (Dp), porosidade total (Pot), micro e macroporosidades (Mip e Map, respectivamente) não apresentaram diferenças estatísticas significativas que indicassem a influência dos sistemas de manejo testados (Quadro 2). No entanto, o tráfego de máquinas e de implementos agrícolas tem sido associado a alterações nessas propriedades (Tormena et al., 1998; Klein \& Libardi, 2002). Provavelmente, a condição textural do solo da área e a baixa intensidade de movimentação na superfície de alguns tratamentos

Quadro 2. Condutividade hidráulica (CH), densidade das partículas (Dp), porosidade total (Pot), microporosidade (Mip) e macroporosidade (Map) em um Argissolo Acinzentado cultivado com cajueiro anão precoce CCP 76 sob diferentes sistemas de manejo, nas profundidades de 0-10 e 20-30 cm, no ano de 2002, no município de Pacajus-CE

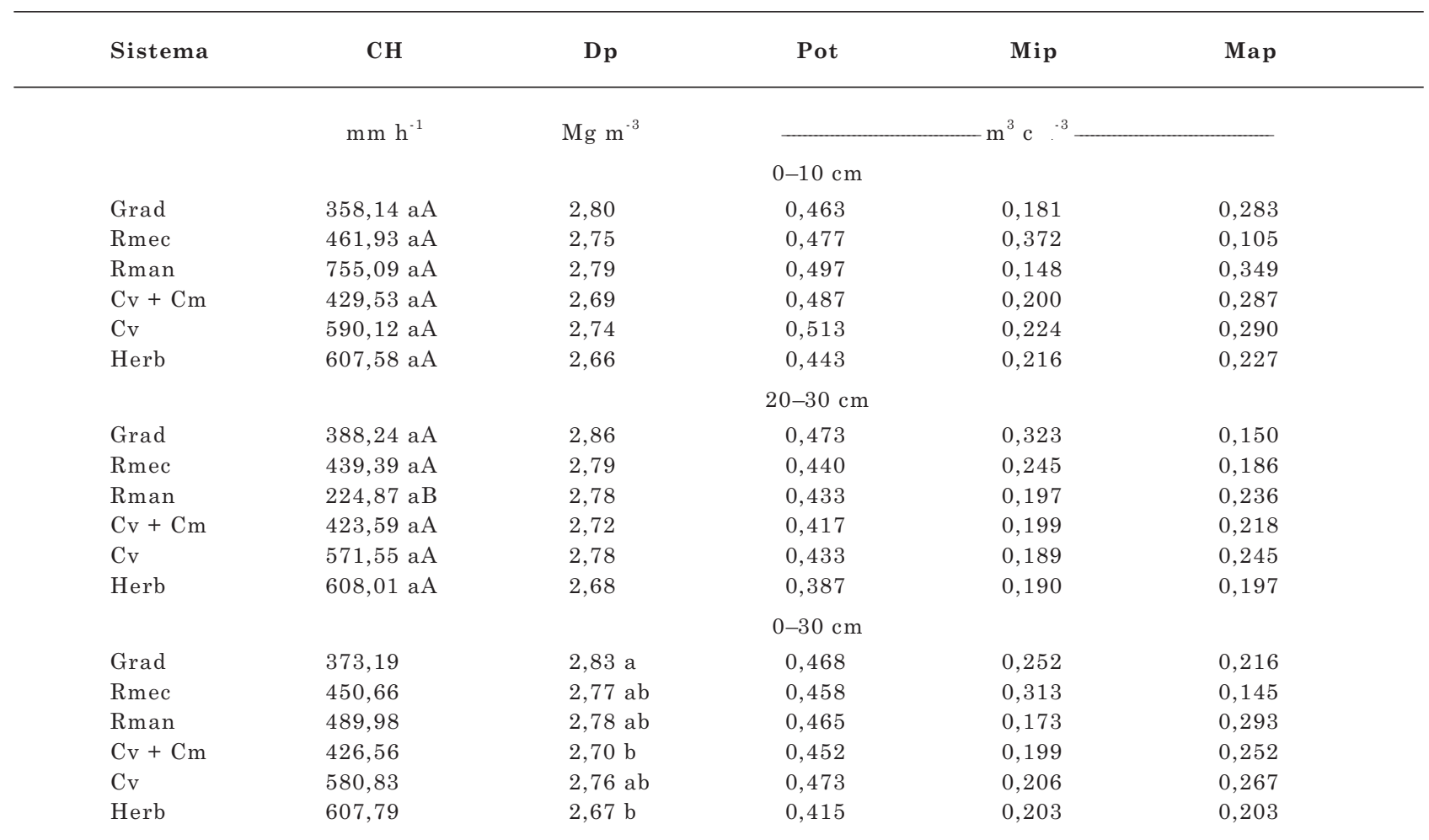

Médias seguidas de mesmas letras minúsculas nas colunas, comparando os tratamentos dentro de cada profundidade isoladamente, não diferem entre si pelo teste de Tukey a $5 \%$. Grad: gradagem + coroamento; Rmec: roçagem mecânica + coroamento; Rman: roçagem manual + coroamento; Cv: cobertura vegetal + coroamento; $\mathrm{Cv}+\mathrm{Cm}$ : cobertura vegetal + cobertura morta; Herb: aplicação de herbicida. 
sejam as razões da não-alteração das propriedades avaliadas. Provavelmente possam contribuir também a presença e o acúmulo temporal de raízes de plantas espontâneas, pois, em alguns tratamentos, a biomassa vegetal da parte aérea é retirada, permanecendo as raízes, as quais se renovam e degradam com o tempo, contrapondo as tendências de adensamento e, ou, compactação em superfície ou subsuperfície.

Ao avaliar os valores médios de Dp (camada de 0-30 cm), no entanto, constata-se que esta foi afetada pelos diferentes sistemas de manejo, sendo que a maior média foi alcançada pelo tratamento com a Grad. De acordo com Reichardt (1975), esta variável praticamente não é influenciada por alterações no manejo, mas está relacionada à constituição mineralógica e ao conteúdo de MO. Provavelmente, o maior valor de Dp ocasionado pela Grad pode estar relacionado ao conteúdo de MO presente nesse solo. Resultados semelhantes a este foram apresentados por Beutier et al. (2001), onde demonstraram que a Dp foi superior em sistems de preparo convencional com grade e com arado de disco na profundidade de $0-5 \mathrm{~cm}$ e relacionou estes valores aos menores teores de MO.

A resistência à penetração $(\mathrm{RP})$ variou de acordo com a umidade do solo entre os diferentes sistemas de manejo (Figura 1). De modo geral, os valores estiveram acima de $2 \mathrm{MPa}$, considerado um valor impeditivo ao crescimento e funcionamento do sistema radicular das plantas (Taylor et al., 1966), embora alguns autores indiquem que esse limite possa estar em torno de 1, 2, 3,5 e $5 \mathrm{MPa}$ (Canarache, 1990; Merotto Jr. \& Mundstock, 1999). O sistema Rman foi o que apresentou o maior valor de RP na profundidade de $20 \mathrm{~cm}(9,8 \mathrm{MPa})$, ocorrendo aumento gradual da RP com a profundidade até atingir $20 \mathrm{~cm}$. Esse incremento não esteve associado à diminuição da umidade, que se manteve relativamente constante (Figura 1). Isso pode ter ocorrido devido à maior proporção de raízes nessa profundidade, que, segundo Genro Junior et al. (2004), preenchem grande parte do sistema poroso do solo, aumentando-lhe a rigidez. Para enfatizar esse argumento, não houve aumento significativo da densidade do solo, o que poderia ser um fator-chave no aumento da RP, como indicam Klein \& Libardi (2002).

A densidade do solo (Ds) não foi influenciada pelos sistemas de manejo (Quadro 3). Os valores obtidos situaram-se na faixa de $1,55 \mathrm{Mg} \mathrm{m}^{-3}$, comum a solos de textura arenosa. Houve tendência de menores valores de Ds, na camada de $0-5 \mathrm{~cm}$, naqueles sistemas que favoreceram o maior aporte de matéria orgânica (Rman, $\mathrm{Cv}+\mathrm{Cm}$ e $\mathrm{Cv}$ ), corroborando os resultados de Beutler et al. (2001) e Falleiro et al. (2003).

RESISTÊNCIA À PENETRAÇÃO (MPa)
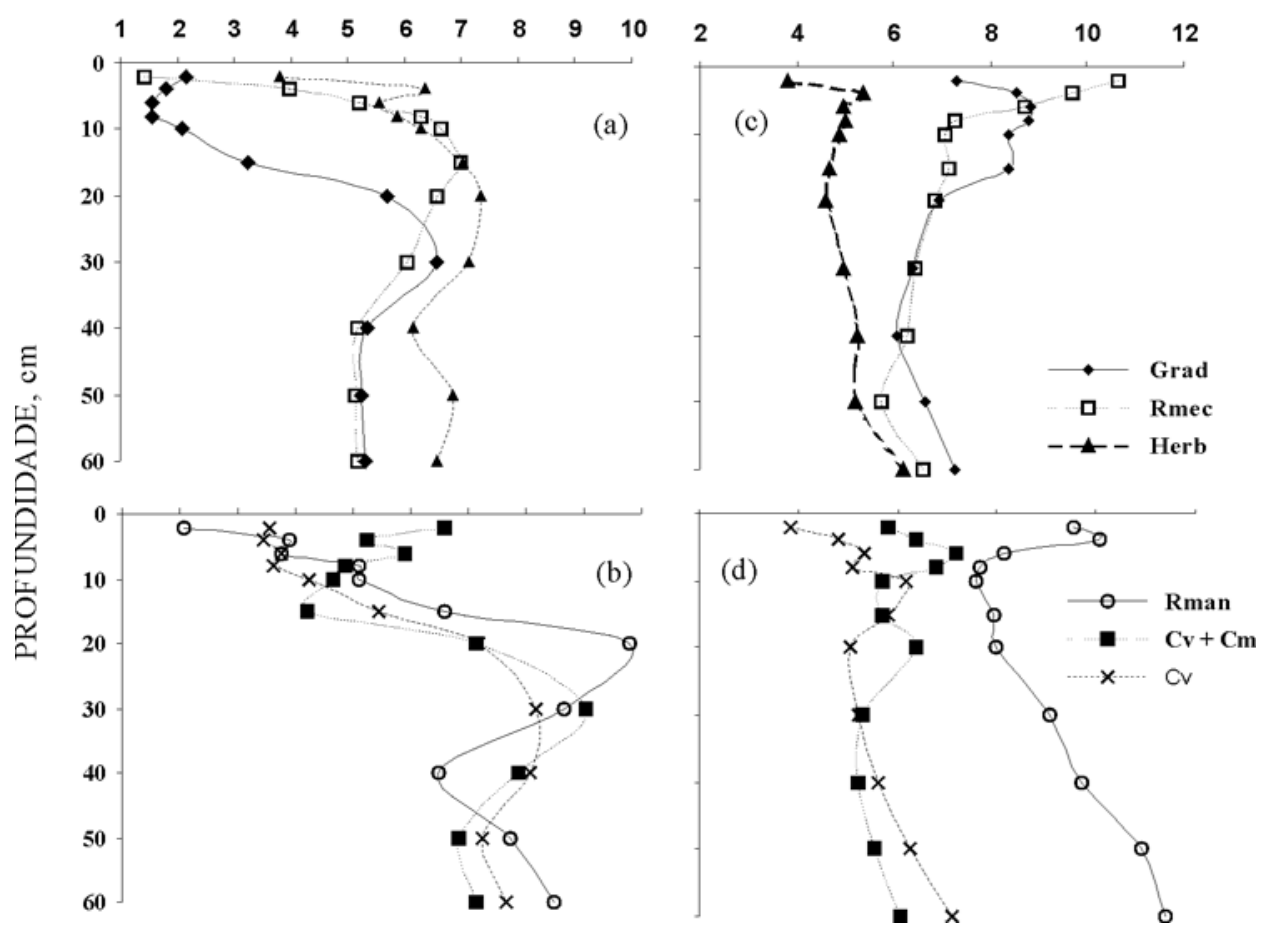

Figura 1. Curvas de resistência à penetração (a e b) e umidade (c e d) de um Argissolo submetido a diferentes sistemas de manejo com a cultura do cajueiro anão precoce, no município de Pacajus, CE. Grad: gradagem + coroamento; Rmec: roçagem mecânica + coroamento; Rman: roçagem manual + coroamento; Cv: cobertura vegetal + coroamento; $\mathrm{Cv}+\mathrm{Cm}$ : cobertura vegetal + cobertura morta; Herb: aplicação de herbicida (média de três repetições). 


\section{Propriedades químicas do solo}

Não houve diferenças significativas entre os sistemas de manejo para as variáveis químicas avaliadas, considerando os três anos de condução do experimento (Quadro 4). Em todos os sistemas de manejo ocorreu tendência de incremento de matéria orgânica (MO) com o tempo. Observa-se que os sistemas Rman

Quadro 3. Densidade em um Argissolo Acinzentado cultivado com cajueiro anão precoce CCP 76, sob diferentes sistemas de manejo

\begin{tabular}{|c|c|c|c|c|c|c|}
\hline \multirow{2}{*}{ Profundidade } & \multicolumn{6}{|c|}{ Sistema } \\
\hline & Grad & Rmec & Rman & $\mathrm{Cv}+\mathrm{Cm}$ & $\mathrm{Cv}$ & Herb \\
\hline $\mathrm{cm}$ & \multicolumn{6}{|c|}{ Densidade, $\mathrm{Mg} \mathrm{m}^{-3}$} \\
\hline $0-5$ & 1,48 & 1,40 & 1,33 & 1,37 & 1,25 & 1,50 \\
\hline $5-10$ & 1,52 & 1,47 & 1,46 & 1,40 & 1,41 & 1,49 \\
\hline $10-15$ & 1,47 & 1,49 & 1,51 & 1,44 & 1,53 & 1,46 \\
\hline $15-20$ & 1,57 & 1,57 & 1,55 & 1,58 & 1,62 & 1,55 \\
\hline $20-25$ & 1,51 & 1,57 & 1,61 & 1,58 & 1,59 & 1,65 \\
\hline $25-30$ & 1,51 & 1,55 & 1,52 & 1,59 & 1,55 & 1,61 \\
\hline $30-35$ & 1,60 & 1,57 & 1,58 & 1,60 & 1,59 & 1,53 \\
\hline $35-40$ & 1,58 & 1,55 & 1,53 & 1,58 & 1,66 & 1,58 \\
\hline $40-45$ & 1,59 & 1,58 & 1,49 & 1,57 & 1,57 & 1,61 \\
\hline $45-50$ & 1,58 & 1,56 & 1,57 & 1,56 & 1,54 & 1,54 \\
\hline $50-55$ & 1,69 & 1,57 & 1,57 & 1,54 & 1,60 & 1,59 \\
\hline $55-60$ & 1,59 & 1,56 & 1,56 & 1,61 & 1,60 & 1,61 \\
\hline
\end{tabular}

Grad: gradagem + coroamento; Rmec: roçagem mecânica + coroamento; Rman: roçagem manual + coroamento; Cv: cobertura vegetal + coroamento; $\mathrm{Cv}+\mathrm{Cm}$ : cobertura vegetal + cobertura morta; Herb: aplicação de herbicida.

Quadro 4. Propriedades químicas de um Argissolo Acinzentado cultivado com cajueiro anão precoce CCP 76 sob diferentes sistemas de manejo, nas profundidades de 0-20 e 20-40 cm, avaliadas nos anos de 1998, 2000 e 2002, no município de Pacajus-CE (média de três repetições e de duas profundidades)

\begin{tabular}{|c|c|c|c|c|c|c|c|c|c|c|c|}
\hline Sistema & pH $(1: 2,5)$ & MO & $P$ disp & $\mathrm{Ca}^{2+}$ & $\mathbf{M g}^{2+}$ & $\mathbf{K}^{+}$ & $\mathrm{Al}^{3+}$ & $\mathbf{H}+\mathbf{A l}$ & $\mathbf{T}$ & $\mathbf{S}$ & V \\
\hline & $\mathrm{H}_{2} \mathrm{O}$ & $\mathrm{g} \mathrm{kg}-1$ & $\mathrm{mg} \mathrm{kg} \cdot 1$ & & 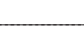 & $-\mathrm{c}$ & $\mathrm{ol}_{\mathrm{c}} \mathrm{kg}$ & & 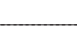 & - & $\%$ \\
\hline \multicolumn{12}{|c|}{1998} \\
\hline Grad & 4,93 & 4,18 & 5,30 & 0,45 & 0,32 & 0,08 & 0,08 & 0,80 & 1,75 & 0,93 & 52,50 \\
\hline Rmec & 5,12 & 3,48 & 4,30 & 0,35 & 0,28 & 0,07 & 0,07 & 0,82 & 1,60 & 0,80 & 49,17 \\
\hline $\mathrm{Rman}$ & 5,13 & 5,63 & 5,80 & 0,70 & 0,40 & 0,09 & 0,09 & 1,10 & 2,40 & 1,33 & 53,33 \\
\hline $\mathrm{Cv}+\mathrm{Cm}$ & 5,13 & 5,40 & 5,20 & 0,65 & 0,33 & 0,08 & 0,08 & 0,93 & 2,05 & 1,13 & 54,83 \\
\hline $\mathrm{Cv}$ & 4,97 & 6,33 & 7,20 & 0,58 & 0,37 & 0,09 & 0,09 & 1,07 & 2,18 & 1,13 & 51,17 \\
\hline Herb & 5,03 & 5,13 & 5,50 & 0,57 & 0,40 & 0,14 & 0,14 & 0,96 & 2,10 & 1,13 & 52,17 \\
\hline \multicolumn{12}{|c|}{2000} \\
\hline Grad & 4,47 & 4,74 & 3,50 & 0,47 & 0,33 & 0,23 & 0,23 & 1,10 & 2,00 & 0,90 & 44,33 \\
\hline Rmec & 4,53 & 3,43 & 2,70 & 0,48 & 0,35 & 0,30 & 0,30 & 1,02 & 2,08 & 1,03 & 48,50 \\
\hline Rman & 4,45 & 8,02 & 3,20 & 0,67 & 0,40 & 0,29 & 0,29 & 1,34 & 2,63 & 2,33 & 47,00 \\
\hline $\mathrm{Cv}+\mathrm{Cm}$ & 4,72 & 4,77 & 3,00 & 0,40 & 0,28 & 0,22 & 0,22 & 1,10 & 1,87 & 0,75 & 40,50 \\
\hline $\mathrm{Cv}$ & 4,42 & 4,36 & 5,00 & 0,58 & 0,42 & 0,24 & 0,24 & 0,99 & 2,15 & 1,15 & 53,83 \\
\hline Herb & 4,08 & 8,15 & 3,50 & 0,47 & 0,27 & 0,23 & 0,23 & 1,13 & 2,00 & 0,85 & 42,50 \\
\hline \multicolumn{12}{|c|}{2002} \\
\hline Grad & 5,25 & 8,38 & 3,31 & 0,74 & 0,08 & 0,09 & 0,09 & 0,11 & 1,23 & 1,13 & 91,01 \\
\hline Rmec & 5,84 & 8,13 & 2,66 & 0,84 & 0,17 & 0,09 & 0,09 & 0,18 & 1,54 & 1,36 & 87,60 \\
\hline Rman & 5,50 & 10,73 & 3,96 & 1,02 & 0,30 & 0,19 & 0,19 & 0,18 & 2,15 & 1,97 & 90,56 \\
\hline $\mathrm{Cv}+\mathrm{Cm}$ & 5,29 & 8,52 & 5,88 & 0,77 & 0,14 & 0,11 & 0,11 & 0,16 & 1,41 & 1,24 & 88,40 \\
\hline $\mathrm{Cv}$ & 5,32 & 10,19 & 5,79 & 0,88 & 0,18 & 0,10 & 0,10 & 0,17 & 1,76 & 1,39 & 90,32 \\
\hline Herb & 4,95 & 9,90 & 6,06 & 0,72 & 0,09 & 0,09 & 0,09 & 0,11 & 1,24 & 1,14 & 87,35 \\
\hline
\end{tabular}

Grad: gradagem + coroamento; Rmec: roçagem mecânica + coroamento; Rman: roçagem manual + coroamento; Cv: cobertura vegetal + coroamento; $\mathrm{Cv}+\mathrm{Cm}$ : cobertura vegetal + cobertura morta; Herb: aplicação de herbicida. 
e Herb foram os que promoveram os maiores aumentos nos níveis de MO entre os anos de 1998 e 2000. Já entre 2000 e 2002, além do Rman, destacou-se também o sistema $\mathrm{Cv}+\mathrm{Cm}$ como aqueles que promoveram os maiores incrementos nos teores de MO no solo. Essa resposta pode estar associada, primeiramente, ao aumento de biomassa vegetal de acordo com a idade da planta, gerando maior aporte de resíduos orgânicos provenientes de folhas e ramos em superfície. Aliado a isso, as maiores adições devem estar relacionadas também à não-remoção dos resíduos orgânicos deixados sobre a copa, como no caso do sistema Herb, que não realizou o coroamento, além da própria adição de material no sistema com cobertura vegetal e, ou, cobertura morta. Essa resposta não é a mesma daquela encontrada no sistema Grad, que, em vez de favorecer acúmulo, promoveu mineralização mais acelerada da MO com o intenso revolvimento do solo (Bayer \& Mielniczuk, 1997).

Os teores de soma de bases (S) encontrados estão relacionados principalmente com os aumentos nos teores de $\mathrm{MO}$ ao longo dos anos, além da influência das adubações químicas realizadas anualmente. Os maiores aumentos foram observados no sistema
Rman, confirmando a influência positiva da MO no aumento da fertilidade do solo. Em adição, este mesmo sistema apresentou maiores valores de capacidade de troca de cátions (T) em relação aos demais. Os maiores valores absolutos para a saturação por bases (V) ocorreram no ano de 2002 , variando de 87 a $91 \%$ entre os sistemas de manejo. O expressivo aumento do valor V entre os anos de 2000 e 2002 possivelmente tenha ocorrido em razão dos aumentos dos teores de MO. No entanto, não se descarta o efeito residual das adubações.

\section{Fracionamento de fósforo}

Analisando a extração seqüencial de P (Quadro 5), nota-se que os maiores teores foram obtidos pela extração realizada em meio ácido, ou seja, houve predomínio das formas mais lábeis de $\mathrm{P}$ no solo entre os diferentes sistemas de manejo. A extração ácida solubiliza formas de $\mathrm{P}$ ligadas a $\mathrm{Ca}^{2+}$ e $\mathrm{Mg}^{2+}$, enquanto, na extração básica, as formas de menor labilidade estão associadas aos íons $\mathrm{Fe}^{2+}$ e $\mathrm{Al}^{3+}$ (Novais \& Smyth, 1999), como também podem formar complexos estáveis com as substâncias húmicas do solo (Passos, 2000).

Quadro 5. Teores de fósforo total (Pt), inorgânico (Pi) e orgânico (Po) extraídos em meio ácido $\left(\mathrm{H}^{+}\right)$e básico $\left(\mathrm{OH}^{-}\right)$em um Argissolo Acinzentado cultivado com cajueiro anão precoce CCP 76, sob diferentes sistemas de manejo, nas profundidades de 0-6, 6-15, 15-30 e 30-60 cm, no município de Pacajus-CE

\begin{tabular}{|c|c|c|c|c|c|c|c|c|}
\hline \multirow{2}{*}{ Variável } & \multirow{2}{*}{ Profundidade } & \multicolumn{6}{|c|}{ Sistema de manejo } & \multirow{2}{*}{ Média } \\
\hline & & Grad & Rmec & Rman & $\mathrm{Cv}$ & $\mathrm{Cv}+\mathrm{Cm}$ & Herb & \\
\hline \multicolumn{9}{|c|}{$\mathrm{cm}$} \\
\hline \multirow{5}{*}{$\begin{array}{l}\mathrm{Pt}\left(\mathrm{H}^{+}\right) \\
\mathrm{mg} \mathrm{kg}^{-1}\end{array}$} & $0-6$ & 11,91 & 19,56 & 29,88 & 27,21 & 31,79 & 28,74 & $24,84 \mathrm{~A}$ \\
\hline & $6-15$ & 19,56 & 24,91 & 25,29 & 29,88 & 30,26 & 29,88 & $26,63 \mathrm{~A}$ \\
\hline & $15-30$ & 11,15 & 13,06 & 31,79 & 18,41 & 19,94 & 23,76 & $19,68 \mathrm{AB}$ \\
\hline & $30-60$ & 10,38 & 6,18 & 23,38 & 18,03 & 20,71 & 18,41 & $16,18 \mathrm{~B}$ \\
\hline & $0-60$ & $13,25 \mathrm{c}$ & $15,92 \mathrm{bc}$ & 27,58 a & $23,38 \mathrm{ab}$ & $25,67 \mathrm{a}$ & 25,19 a & 21,83 \\
\hline \multirow{5}{*}{$\begin{array}{l}\mathrm{Pi}\left(\mathrm{H}^{+}\right) \\
\mathrm{mg} \mathrm{kg}^{-1}\end{array}$} & $0-6$ & $8,26 \mathrm{aAB}$ & $13,11 \mathrm{aA}$ & $15,36 \mathrm{aA}$ & $11,81 \mathrm{aA}$ & $12,81 \mathrm{aA}$ & 9,99 a B & 11,89 \\
\hline & $6-15$ & $11,07 \mathrm{bA}$ & $5,44 \mathrm{bB}$ & $10,94 \mathrm{bAB}$ & $12,51 \mathrm{bA}$ & $11,31 \mathrm{bAB}$ & $25,63 \mathrm{aA}$ & 12,81 \\
\hline & $15-30$ & $7,29 \mathrm{aAB}$ & $2,70 \mathrm{aB}$ & $6,74 \mathrm{aB}$ & $9,99 \mathrm{aA}$ & $5,90 \mathrm{aBC}$ & $6,94 \mathrm{aBC}$ & 6,59 \\
\hline & $30-60$ & $3,92 \mathrm{aB}$ & $1,90 \mathrm{aB}$ & $6,11 \mathrm{aB}$ & $4,12 \mathrm{aB}$ & $3,90 \mathrm{aC}$ & $3,57 \mathrm{aC}$ & 3,92 \\
\hline & $0-60$ & 7,63 & 5,78 & 9,78 & 9,60 & 8,48 & 11,53 & 8,80 \\
\hline \multirow{5}{*}{$\begin{array}{l}\mathrm{Po}\left(\mathrm{H}^{+}\right) \\
\mathrm{mg} \mathrm{kg}^{-1}\end{array}$} & $0-6$ & 3,65 & 6,45 & 14,51 & 15,39 & 18,98 & 18,74 & 12,95 \\
\hline & $6-15$ & 8,48 & 19,47 & 14,35 & 17,37 & 18,95 & 6,02 & 14,10 \\
\hline & $15-30$ & 3,85 & 10,35 & 25,05 & 8,42 & 14,04 & 16,82 & 13,08 \\
\hline & $30-60$ & 6,45 & 4,27 & 17,27 & 13,90 & 16,80 & 15,19 & 12,31 \\
\hline & $0-60$ & $5,60 \mathrm{~b}$ & $10,13 \mathrm{ab}$ & 17,79 a & $13,77 \mathrm{ab}$ & 17,19 a & $14,19 \mathrm{ab}$ & 13,12 \\
\hline \multirow{5}{*}{$\begin{array}{l}\mathrm{Pt}\left(\mathrm{OH}^{-}\right) \\
\mathrm{mg} \mathrm{kg}^{-1}\end{array}$} & $0-6$ & $7,18 \mathrm{abB}$ & 9,86 aA & $3,26 \mathrm{cA}$ & $4,70 \mathrm{bcA}$ & $4,70 \mathrm{bcA}$ & $5,53 \mathrm{bcA}$ & 5,87 \\
\hline & $6-15$ & $6,97 \mathrm{abB}$ & 8,83 aA & $3,88 \mathrm{cA}$ & $4,29 \mathrm{bcA}$ & 5,94 abcA & $5,12 \mathrm{bcA}$ & 5,83 \\
\hline & $15-30$ & 9,86 aA & $0,79 \mathrm{cB}$ & $3,47 \mathrm{bA}$ & $4,91 \mathrm{bA}$ & $5,32 \mathrm{bA}$ & $4,91 \mathrm{bA}$ & 4,87 \\
\hline & $30-60$ & $8,41 \mathrm{aAB}$ & $0,99 \mathrm{cB}$ & $2,85 \mathrm{bA}$ & $4,29 \mathrm{bA}$ & $5,32 \mathrm{bA}$ & $4,70 \mathrm{bA}$ & 4,42 \\
\hline & $0-60$ & 8,10 & 5,11 & 3,36 & 4,54 & 5,32 & 5,06 & 5,24 \\
\hline \multirow{5}{*}{$\begin{array}{l}\mathrm{Pi}\left(\mathrm{OH}^{-}\right) \\
\mathrm{mg} \mathrm{kg}^{-1}\end{array}$} & $0-6$ & 0,64 & 0,45 & 0,95 & 0,70 & 0,79 & 0,82 & 0,72 \\
\hline & $6-15$ & 0,61 & 0,51 & 1,66 & 0,67 & 0,80 & 0,62 & 0,81 \\
\hline & $15-30$ & 0,74 & 0,41 & 1,11 & 0,80 & 0,72 & 0,70 & 0,74 \\
\hline & $30-60$ & 0,54 & 0,21 & 1,19 & 0,66 & 0,72 & 0,67 & 0,66 \\
\hline & $0-60$ & $0,63 \mathrm{ab}$ & $0,39 \mathrm{~b}$ & $1,22 \mathrm{a}$ & $0,70 \mathrm{ab}$ & $0,75 \mathrm{ab}$ & $0,70 \mathrm{ab}$ & 0,73 \\
\hline \multirow{5}{*}{$\begin{array}{l}\text { Po }\left(\mathrm{OH}^{-}\right) \\
\mathrm{mg} \mathrm{kg}^{-1}\end{array}$} & $0-6$ & $6,54 \mathrm{abB}$ & 9,19 aA & $2,31 \mathrm{cA}$ & $4,00 \mathrm{bcA}$ & $3,92 \mathrm{bcA}$ & $4,71 \mathrm{bcA}$ & 5,11 \\
\hline & $6-15$ & $6,37 \mathrm{abB}$ & 8,32 aA & $2,22 \mathrm{cA}$ & $3,62 \mathrm{bcA}$ & $5,14 \mathrm{bA}$ & $4,50 \mathrm{bcA}$ & 5,02 \\
\hline & $15-30$ & $9,12 \mathrm{aA}$ & $0,38 \mathrm{cB}$ & $2,36 \mathrm{bcA}$ & $4,11 \mathrm{bA}$ & $4,60 \mathrm{bA}$ & $4,21 \mathrm{bA}$ & 4,13 \\
\hline & $30-60$ & $7,87 \mathrm{aAB}$ & $0,78 \mathrm{~dB}$ & $1,66 \mathrm{cdA}$ & $3,64 \mathrm{bcA}$ & $4,60 \mathrm{bA}$ & $4,04 \mathrm{bcA}$ & 3,76 \\
\hline & $0-60$ & 7,47 & 4,66 & 2,13 & 3,84 & 4,56 & 4,36 & 4,50 \\
\hline
\end{tabular}

Grad: gradagem + coroamento; Rmec: roçagem mecânica + coroamento; Rman: roçagem manual + coroamento; Cv: cobertura vegetal + coroamento; $\mathrm{Cv}+\mathrm{Cm}$ : cobertura vegetal + cobertura morta; Herb: aplicação de herbicida. 
A fração orgânica $(\mathrm{Po})$ foi aquela que apresentou maior participação no conteúdo total do $\mathrm{P}$ lábil $\left(\mathrm{Pt}\left(\mathrm{H}^{+}\right)\right)$ (Figura 2). Os valores médios de $\mathrm{Po}\left(\mathrm{H}^{+}\right)$variaram de 5,60 a $17,79 \mathrm{mg} \mathrm{kg}^{-1}$ entre os sistemas de manejo. O sistema $\mathrm{Cv}+\mathrm{Cm}$ foi o que apresentou maior percentual de proporção dos teores de $\mathrm{Po}\left(\mathrm{H}^{+}\right)$sobre os de $\mathrm{Pt}\left(\mathrm{H}^{+}\right)$, representando cerca de $67 \%$ do $\mathrm{Pt}\left(\mathrm{H}^{+}\right)$. De modo geral, os tratamentos que favoreceram o maior aporte de $\mathrm{MO}$ apresentaram valores de $\mathrm{Po}\left(\mathrm{H}^{+}\right)$mais elevados em relação aos sistemas mais intensivos, como o Grad.

As maiores proporções da fração $\mathrm{Po}\left(\mathrm{H}^{+}\right)$em relação à fração inorgânica $(\mathrm{Pi})$ aponta a importância da contribuição dessa fração para a manutenção da disponibilidade de $\mathrm{P}$ no solo. Em solos mais intemperizados, o $\mathrm{P}$ disponível pode estar intimamente associado à fração orgânica (Guerra et al., 1996). Em solos de textura arenosa, como o utilizado no presente estudo, a contribuição da fração orgânica passa a ser de fundamental importância, atuando como mecanismo regulador da disponibilidade de $\mathrm{P}$ na solução, visto que, de modo geral, os níveis de $\mathrm{P}$ disponível são relativamente baixos nestes solos. Nesse sentido, sistemas de manejo que favoreçam aporte de $\mathrm{MO}$ e menor revolvimento do solo, como o $\mathrm{Cv}$ e, ou, $\mathrm{Cv}+\mathrm{Cm}$, tornam-se alternativas para o manejo sustentável não somente para a disponibilidade de $\mathrm{P}$, mas também de outros nutrientes essenciais à cultura do caju.

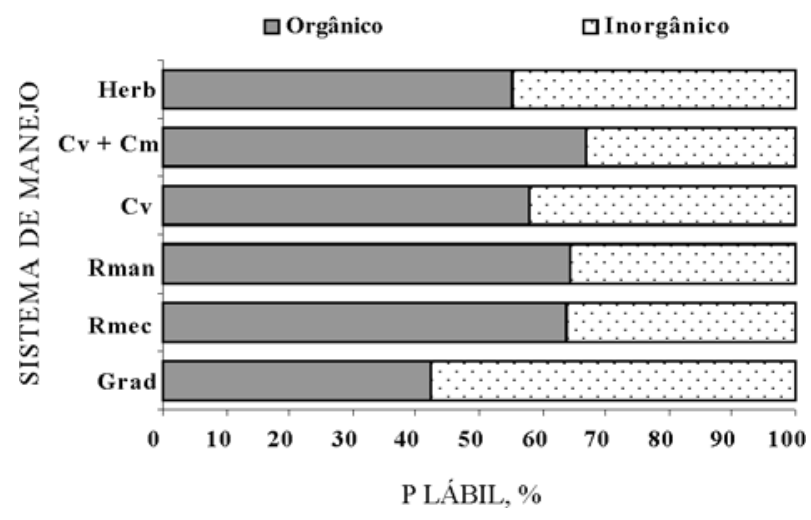

Figura 2. Distribuição percentual das frações lábeis do fósforo obtidas pelo fracionamento em meio ácido, determinado em amostras de um Argissolo Acinzentado cultivado com a cultura do cajueiro anão precoce, sob diferentes sistemas de manejo, no município de Pacajus, CE. Grad: gradagem + coroamento; Rmec: roçagem mecânica + coroamento; Rman: roçagem manual + coroamento; $\mathrm{Cv}$ : cobertura vegetal + coroamento; $\mathrm{Cv}+\mathrm{Cm}$ : cobertura vegetal + cobertura morta; Herb: aplicação de herbicida (média de três repetições).

\section{Produtividade de castanha de caju, altura e diâmetro de copa dos cajueiros}

Apesar da não-uniformidade dos resultados de produtividade de castanha de caju, há tendência de estabilização dos rendimentos, comparando-se com as produtividades médias obtidas nos campos comerciais da cultura, que foram aproximadamente de $1.368 \mathrm{~kg} \mathrm{ha}^{-1}$ (Araújo \& Silva, 1995). Analisando os resultados de produtividade (Quadro 6), o sistema Herb foi aquele que se destacou em todos os anos avaliados, apresentando os maiores índices em relação aos demais sistemas. Houve alternância entre os sistemas de manejo mais produtivos após o Herb. O Rman apresentou maiores índices nos anos de 1999 e 2000, seguido dos sistemas Grad e Cv em 2001 e 2002.

Os maiores rendimentos da castanha de caju no sistema Herb, o qual utiliza o controle de plantas invasoras pelo uso de herbicidas, não devem estar relacionados diretamente à utilização deste insumo, mas indiretamente, pela presença de MO na área, contribuindo com a maior disponibilidade de nutrientes. A não-adoção do coroamento-caracterizado pela limpeza superficial dos restos culturais e eliminação de plantas espontâneas sob a copa do cajueiro - contribuiu com o acúmulo temporal de MO no solo, refletindo em aumento dos níveis de produtividade. Isso deve estar associado aos efeitos integrados da redução da temperatura de solo e da conservação de umidade sob a copa das árvores, como salientado por Despersch et al. (1985) e Vieira et al. (1991). Aliado a isso, tem-se uma maior ciclagem de nutrientes, advindos de um aporte efetivo de material vegetal, além da acumulação de restos de fertilizantes. Morais et al. (2004), em avaliação específica conduzida no mesmo experimento do presente estudo, constataram que a não-eliminação das plantas espontâneas e, ou, a remoção superficial de restos culturais favoreceram melhores condições de umidade e ciclagem de nutrientes no solo.

Nos demais sistemas, os níveis de produtividade mantiveram-se semelhantes durante os anos de avaliação, embora algumas exceções tenham ocorrido em alguns anos de forma isolada (Quadro 6).

As variáveis biométricas, como altura e diâmetro de copa, mostraram comportamento semelhante aos dados de produtividade da castanha de caju durante os anos de avaliação, apresentando resultados esperados, como aumento da altura e das copas das plantas com o tempo. No cultivo do cajueiro anão precoce, as podas de limpeza e de condução são realizadas anualmente. Mesmo assim, essa certa uniformização das árvores não impediu que as diferenças entre os tratamentos fossem ressaltadas, podendo estabelecer a relação do comportamento entre os níveis de produtividade de castanha de caju e os parâmetros biométricos avaliados. 
Quadro 6. Produtividades de castanha de caju, diâmetros da copa nas direções norte-sul e leste-oeste e altura de plantas de cajueiro anão precoce CCP 76 cultivado sob diferentes sistemas de manejo, nos anos de 1999, 2000, 2001 e 2002, no município de Pacajus-CE

\begin{tabular}{|c|c|c|c|c|c|c|c|c|}
\hline \multirow[b]{2}{*}{ Variável } & \multirow[b]{2}{*}{ Ano } & \multicolumn{6}{|c|}{ Sistemas de manejo } & \multirow[b]{2}{*}{ CV (\%) (1) } \\
\hline & & Grad & Rmec & Rman & $\mathrm{Cv}$ & $\mathrm{Cv}+\mathrm{Cm}$ & Herb & \\
\hline \multirow[t]{4}{*}{ Produtividade $\left(\mathrm{kg} \mathrm{ha}^{-1}\right)$} & 1999 & $79,14 \mathrm{~b}$ & 66,22 b & $133,15 \mathrm{ab}$ & $54,21 \mathrm{~b}$ & $78,94 \mathrm{~b}$ & $240,21 \mathrm{a}$ & 40,84 \\
\hline & 2000 & $129,50 \mathrm{~b}$ & $121,34 \mathrm{~b}$ & $262,75 \mathrm{ab}$ & $130,78 \mathrm{~b}$ & $114,36 \mathrm{~b}$ & $366,15 \mathrm{a}$ & 37,06 \\
\hline & 2001 & $474,94 \mathrm{ab}$ & $301,74 \mathrm{~b}$ & $423,15 \mathrm{~b}$ & $440,72 \mathrm{ab}$ & $433,37 \mathrm{~b}$ & $723,90 \mathrm{a}$ & 21,96 \\
\hline & 2002 & $948,37 \mathrm{ab}$ & $704,69 \mathrm{~b}$ & $976,87 \mathrm{ab}$ & $939,23 \mathrm{ab}$ & $949,99 \mathrm{ab}$ & $1.259,92 \mathrm{a}$ & 18,12 \\
\hline \multirow[t]{4}{*}{ Altura (m) } & 1999 & $1,25 \mathrm{ab}$ & $1,15 \mathrm{~b}$ & $1,39 \mathrm{ab}$ & $1,16 \mathrm{~b}$ & $1,18 \mathrm{~b}$ & $1,47 \mathrm{a}$ & 6,78 \\
\hline & 2000 & $1,47 \mathrm{a}$ & $1,58 \mathrm{~b}$ & $2,10 \mathrm{a}$ & $1,84 \mathrm{ab}$ & $1,82 \mathrm{ab}$ & $2,17 \mathrm{a}$ & 6,98 \\
\hline & 2001 & $2,17 \mathrm{ab}$ & $1,85 \mathrm{~b}$ & $2,25 \mathrm{a}$ & $2,16 \mathrm{ab}$ & $2,12 \mathrm{ab}$ & $2,42 \mathrm{a}$ & 5,31 \\
\hline & 2002 & $2,75 \mathrm{ab}$ & $2,37 \mathrm{~b}$ & $2,75 \mathrm{ab}$ & 2,85 a & $2,72 \mathrm{ab}$ & $3,07 \mathrm{a}$ & 5,01 \\
\hline \multirow{4}{*}{$\begin{array}{l}\text { Diâmetro da copa, } \\
\text { direção norte-sul (m) }\end{array}$} & 1999 & $2,20 \mathrm{abc}$ & $2,05 \mathrm{bc}$ & $2,50 \mathrm{ab}$ & $1,98 \mathrm{c}$ & $2,01 \mathrm{bc}$ & $2,68 \mathrm{a}$ & 8,02 \\
\hline & 2000 & $3,43 \mathrm{bc}$ & $3,10 \mathrm{c}$ & $4,04 \mathrm{ab}$ & $3,34 \mathrm{c}$ & $3,24 \mathrm{c}$ & $4,20 \mathrm{a}$ & 6,16 \\
\hline & 2001 & $4,26 \mathrm{abc}$ & $3,77 \mathrm{c}$ & $4,54 \mathrm{ab}$ & $4,09 \mathrm{bc}$ & $3,99 \mathrm{bc}$ & $4,80 \mathrm{a}$ & 5,06 \\
\hline & 2002 & $5,55 \mathrm{~b}$ & $4,49 \mathrm{c}$ & $5,46 \mathrm{~b}$ & $5,73 \mathrm{ab}$ & $5,22 \mathrm{bc}$ & $6,39 \mathrm{a}$ & 4,75 \\
\hline \multirow{4}{*}{$\begin{array}{l}\text { Diâmetro da copa, } \\
\text { direção leste-oeste (m) }\end{array}$} & 1999 & $2,15 \mathrm{ab}$ & $2,13 \mathrm{ab}$ & $2,53 \mathrm{a}$ & $1,93 \mathrm{~b}$ & $2,08 \mathrm{ab}$ & $2,67 \mathrm{a}$ & 9,47 \\
\hline & 2000 & $3,48 \mathrm{bc}$ & $3,03 \mathrm{c}$ & $3,92 \mathrm{ab}$ & $3,28 \mathrm{c}$ & $3,21 \mathrm{c}$ & $4,19 \mathrm{a}$ & 5,58 \\
\hline & 2001 & $4,06 \mathrm{bc}$ & $3,68 \mathrm{c}$ & $4,54 \mathrm{ab}$ & $4,07 \mathrm{bc}$ & $3,96 \mathrm{bc}$ & $4,89 \mathrm{a}$ & 5,98 \\
\hline & 2002 & $5,41 \mathrm{ab}$ & $4,29 \mathrm{c}$ & $5,30 \mathrm{~b}$ & $5,66 \mathrm{ab}$ & $5,27 \mathrm{~b}$ & 6,32 a & 5,99 \\
\hline
\end{tabular}

Médias seguidas de mesma letra nas linhas não diferem entre si pelo teste de Tukey a $5 \%$.

${ }^{(1)} \mathrm{CV}$ : coeficiente de variação. Grad: gradagem + coroamento; Rmec: roçagem mecânica + coroamento; Rman: roçagem manual + coroamento; Cv: cobertura vegetal + coroamento; Cv + Cm: cobertura vegetal + cobertura morta; Herb: aplicação de herbicida.

\section{CONCLUSÕES}

1. Os sistemas de manejo estudados promoveram alterações nas propriedades químicas do solo, principalmente naquelas associadas à matéria orgânica do solo, com reflexos positivos nos sistemas que utilizam o herbicida e a roçagem manual e negativos no que envolve a grade de discos.

2. O não-revolvimento superficial do solo e a nãofragmentação da biomassa aérea das plantas espontâneas presentes nas entrelinhas, previstos nos sistemas que utilizam o herbicida e a roçagem manual, constituem-se em estratégias de manejo favoráveis à melhoria da qualidade do solo e à produtividade dos cajueiros.

3. A ausência da prática do coroamento promoveu melhor qualidade do solo e maiores níveis de produtividade de castanha em decorrência do aumento do aporte de matéria orgânica no solo e do não-revolvimento superficial do solo, permitindo inferir que não se trata de uma prática adequada às culturas perenes.

\section{LITERATURA CITADA}

ARAÚJO, J.P. \& SILVA V.V. Cajucultura: modernas técnicas de produção. Fortaleza, Embrapa-CNPAT, 1995. 292p.
ARAÚJO, J.P.P. Situação atual e perspectivas da cajucultura brasileira. Fortaleza, Embrapa, 1990 (Caju Informativo, 3)

BARRETO, A.C. \& FERNANDES, M.F. Cultivo de Gliricidia sepium e Leucena leucocephala em alamedas visando a melhoria dos solos dos tabuleiros costeiros. Pesq. Agropec. Bras., 36:1287-1293, 2001.

BAYER, C. \& MIELNICZUK J. Características químicas do solo afetadas por métodos de preparo e sistemas de cultura. R. Bras. Ci. Solo, 21:105-112, 1997.

BEUTLER, A.N.; SILVA, M.L.N.; CURI, N.; FERREIRA, M.M.; CRUZ, J.C. \& PEREIRA FILHO, I.A. Resistência à penetração e permealidade de Latossolo Vermelho distrófico típico sob sistemas de manejo na região do cerrado. R. Bras. Ci. Solo, 25:167-177, 2001.

BOWMAN, R.A. A sequential extraction procedure with concentrated sulfuric acid and dilute base for soil organic phosphorus. Soil Sci. Soc. Am. J., 53:362-366, 1989.

CANARACHE, A. Penetr - a generalized semi-empirical model estimating soil resistence to penetration. Soil Till. Res., 16:51-70, 1990.

CATTELAN, A.J. \& VIDOR, C. Flutuações na biomassa, atividade e população microbiana do solo, em função de variações ambientais. R. Bras. Ci. Solo, 14:133-142, 1990. 
DESPERSCH, R.; SIDIRAS, N. \& HEINZMANN, F.X. Manejo do solo com coberturas verdes de inverno. Pesq. Agropec. Bras., 20:761-773, 1985.

EMPRESA BRASILEIRA DE PESQUISA AGROPECUÁRIA . EMBRAPA. Manual de métodos de análise de solos. Rio de Janeiro, 1997. 212p.

EMPRESA BRASILEIRA DE PESQUISA AGROPECUÁRIA. - EMBRAPA. Manual de análises químicas de solos, plantas e fertilizantes. Brasília, Embrapa Solos, Embrapa Informática Agropecuária, 1999. 370p.

FALLEIRO, R.M.; SOUZA, C.M.; SILVA, C.S.W.; SEDIYAMA, C.S.; SILVA, A.A. \& FAGUNDES, J.L. Influência dos sistemas de preparo nas propriedades químicas e físicas do solo. R Bras. Ci. Solo, 27:1097-1104, 2003.

FUNDAÇÃO INSTITUTO DE PESQUISA E INFORMAÇÃO DO CEARÁ. - IPLANCE. Perfil básico municipal: Pacajus. Banco de dados agregados. Disponível em: < http:// www.iplance.ce.gov.br/Arquivos/3/1/2000/ PBM_Pacajus.pdf >. Acesso em: 28 jan. 2004.

GENRO JUNIOR, S.A.; REINERT, D.J. \& REICHERT, J.M. Variabilidade temporal da resistência à penetração de um Latossolo argiloso sob semeadura direta com rotação de culturas. R. Bras. Ci. Solo, 28:477-484, 2004.

GUERRA, J.G.M.; ALMEIDA, D.L.; SANTOS, G.A. \& FERNANDES, M.S. Conteúdo de fósforo orgânico em amostras de solos. Pesq. Agropec. Bras., 31:291-299, 1996.

INSTITUTO BRASILEIRO DE GEOGRAFIA E ESTATÍSTICA - IBGE. Perfil básico municipal: Pacajus. Banco de dados agregados. Sistema IBGE de Recuperação Automática SIDRA: Tema Agricultura. Disponível em <http:// www.sidra.ibge.gov.br >. Acesso em: 10 jan. 2004.

KLEIN, V.A. \& LIBARDI, P.L. Densidade e distribuição do diâmetro dos poros de um Latossolo vermelho, sob diferentes sistemas de uso e manejo. R. Bras. Ci. Solo, 26:857-867, 2002.

LEITE, L.F.C.; MENDONÇA, E.S.; NEVES, J.C.L.; MACHADO, P.L.O.A. \& GALVÃO, J.C.C. Estoques totais de carbono orgânico e seus compartimentos em Argissolo sob floresta e sob milho cultivado com adubação mineral e orgânica. R. Bras. Ci. Solo, 27:821-832, 2003.

LIMA, V.P.M.S. Origem e distribuição geográfica. In: LIMA, V.P.M.S., org. A cultura do cajueiro no Nordeste brasileiro. Fortaleza, BNB/ETENE, 1988. 486p. (Estudos Econômicos e Sociais, 35)

LOPES NETO, A. Agroindústria do caju. Fortaleza, Iplance, 1997. 263p.

MEROTTO Jr., A. \& MUNDSTOCK, C.M. Wheat root growth as affected by soil strenght. R. Bras. Ci. Solo, 23:135-143, 1999.
MONREAL, C.M.; ZENTNER, R.P. \& ROBERTSON, J.A. An analysis of soil organic matter dynamics in relation to management, erosion and yield of wheat in long-term crop rotation plots. Can. J. Soil Sci., 77:553-563, 1997.

MORAIS, G.M.; SARAIVA, J.P.B.; FREITAS, H.H.S.; OLIVEIRA, T.S.; MENDONÇA, E.S. \& CRISOSTOMO, L.A. Aporte de matéria orgânica e nutrientes em sistemas de manejo do solo na cultura do cajueiro anão precoce. In: OLIVEIRA, T.S.; MENDONÇA, E.S.; XAVIER, F.A.S.; LIBARDI, P.L. \& ASSIS Jr., R.N. Solo e água: aspectos de uso e manejo com ênfase no semi-árido nordestino. Fortaleza, UFC/UFV/ESALQ/CAPES, 2004. p.156-175.

MURPHY, J. \& RILEY, J.P. A modified single solution method for the determination of phosphate in natural waters. Anal.. Chim. Acta, 27:31-36,1962.

NOVAIS, R.F. \& SMYTH, T.J. Fósforo em solo e planta em condições tropicais.Viçosa, MG, Universidade Federal de Viçosa, 1999. 224p.

PASSOS, R.R. Carbono orgânico e nitrogênio em agregados de Latossolo Vermelho sob duas coberturas vegetais. Viçosa, MG, Universidade Federal de Viçosa, 2000. 89p (Tese de Mestrado)

PAULA. M.B.; ASSIS, R.P.; BAHIA, V.G. \& OLIVEIRA, C.V. Efeito do manejo dos resíduos culturais, adubos verdes, rotação de culturas e aplicação de corretivos nas propriedades físicas e recuperação dos solos. Inf. Agropec., 19:66-70, 1998.

PIMENTEL, C.R.M.; PAULA PESSOA, P.F.A. \& LIMA. L.A.A. Análise estrutural e disponibilidade de tecnologia para a cajucultura brasileira. Fortaleza, Embrapa-CNPAT, 1993. 31p. (Documento, 8)

REICHARDT, K. Processos de transferência no sistema soloplanta-atmosfera. 3.ed. Piracicaba, CENA, Fundação Cargil, 1975. 286p.

TAYLOR, H.M.; ROBERSON, G.M. \& PARKER Jr., J.J. Soil strenght-root penetration relations to medium to coarsetextured soil materials. Soil Sci., 102:18-22, 1966.

TESTA, V.M.; TEIXEIRA, L.A.J. \& MIELNICZUK, J. Características químicas de um Podzólico VermelhoEscuro afetado por sistemas de culturas. R. Bras. Ci. Solo, 16:107-114, 1992.

TORMENA, C.A.; SILVA, A.P. \& LIBARDI, P.L. Caracterização do intervalo hídrico ótimo de um Latossolo roxo sob plantio direto. R. Bras. Ci. Solo, 22:573-581, 1998.

VIEIRA, S.R.; NASCIMENTO, P.C.; SARVASI, F.O.C. \& MOURA, E.G. Umidade e temperatura da camada superficial do solo em função da cobertura morta por resteva de soja em plantio direto. R. Bras. Ci. Solo, 15:219$224,1991$.

YEOMANS, J.C. \& BREMNER, J.M. A rapid and precise method for routine determination of organic carbon in soil. Comm. Soil Sci. Plant Anal., 19:1467-1476, 1988. 